\title{
Accuracy of Multislice Detector Computed Tomography in Staging Borderline Resectable Periampullary Pancreatic Carcinoma
}

\author{
Mohamed Issa, MD; ${ }^{1}$ Marwa Badawi, MD; ${ }^{2}$ Ahmed Shalaby, MD; ${ }^{2}$ Khaled Noureldin, MD² \\ ${ }^{1}$ Department of General Surgery, Prince Charles Hospital, Merthyr Tydfil, Cardiff, UK \\ ${ }^{2}$ Department of General Surgery, Cairo University Hospitals, Egypt
}

\begin{abstract}
Introduction: Periampullary malignancies have poor outcomes. Thus, identifying patients who can benefit from surgery while avoiding risks and possible complications is crucial. This study was designed to determine the accuracy of MDCT in diagnosing resectable tumors.
\end{abstract}

\begin{abstract}
Methodology and results: This retrospective case-control study included 28 randomly selected patients. Patients were divided into group A (preoperative staging via MDCT) and group B (control; staging via laparoscopy and MDCT).MDCT had a $100 \%$ sensitivity and accuracy in identifying signs of irresectability. For borderline staging, MDCT had an accuracy of $62.5 \%$ and $71 \%$ for groups A and B, respectively. MDCT had an overall accuracy of $75 \%$, which decreased to $68.1 \%$ for borderline lesions. Laparoscopy was able to identify occult findings missed by radiological imaging. Three cases in group $A$ and one in group B underwent unnecessary laparotomy, resulting in a false-negative rate of $21 \%$ and $7 \%$, respectively, with an overall rate of $14.2 \%$. The mean predictive value of CT was $100 \%$ and $68.1 \%$ in irresectable and resectable cancers, respectively.
\end{abstract}

Conclusion: Although MDCT is highly accurate for high-stage cancers, in potentially resectable tumors, it's less accurate. We recommend adding other adjuncts to avoid unindicated laparotomies.

Key words: CT scan sensitivity, borderline periampullary pancreatic cancers, pancreatic cancer staging, multi-detector row computed tomography (MDCT).

\section{Introduction}

Periampullary neoplasms originate from the pancreatic head, distal common bile duct, and second part of the duodenum. The preoperative workup aims to differentiate pancreatic origin from nonpancreatic origin lesions while also confirming the preoperative stage and signs of irresectablity and inoperability, which helps with decision making and further management planning. For operable tumors, Whipple's procedure has been the surgery of choice, whereas for irresectable tumors, palliative and supportive therapy has been provided. Throughout our practice, multislice detector computed tomography (MDCT) has been the diagnostic approach of choice in cases with equivocal CT findings or ambiguous signs, with magnetic resonance imaging, endoscopic ultrasonography (EUS), endoscopic retrograde cholangiopancreatography (ERCP), and percutaneous transhepatic cholangiography being added for further diagnosis, biopsy, or treatment (stent insertion for obstructive jaundice). Staging systems based on CT findings have proven to be the most reliable in periampullary pancreatic cancers. Given that the patient's clinical profile, functional status, and laboratory findings are also considered in the management plan, a degree of overlap can be present in the imaging findings for specific pathologies. Evidence-based protocols refer to MDCT as the cornerstone modality for the assessment of periampullary lesions..$^{1-4}$

\section{Materials and methods}

This retrospective study was conducted between January 2017 and March 2018 and included 28 patients who presented to the outpatient clinic at Kasr Alainy, Cairo University Hospitals. All patients were assessed clinically, after which a detailed clinical history was obtained from all patients in whom cachexia, weight loss, and obstructive jaundice were the dominant clinical features. All patients underwent full laboratory investigations (Complete blood count, urea and electrolytes, liver function test, coagulation profile, cross-match, and carbohydrate antigen 19-9). All patients underwent IV contrast-enhanced chest, abdominal, and pelvic CT to diagnose ampullary/periampullary cancer, and the metastatic status of all patients were discussed by the local multidisciplinary team. Our inclusion criteria were as follows: all patients with potentially curable cancers in the periampullary/ ampullary region and those who were medically fit for surgical resection (All preoperative patients were assessed by an anesthetist and cardiopulmonary exercise testing to determine fitness for surgery). Our exclusion criteria were as follows: (1) extraabdominal metastatic spread confirmed by any form of radiological imaging; (2) benign periampullary lesions; (3) pancreatic cancers in the body or tail; (4) patients with local vascular invasion; and (5) frail patients unfit to undergo pancreaticoduodenectomy. After applying the inclusion and exclusion criteria, 28 patients were recruited into this study and 
equally divided into two groups (Groups A and B). Group A underwent MDCT as the primary diagnostic modality, with ERCP being conducted when indicated for tissue biopsies and stenting. Additional EUS was used to assess local invasion of the portal vein and superior mesenteric artery (SMA) when suspected on CT, as well as the lymph nodes in Passaro's triangle. Meanwhile, group B received similar procedures as group $A$ apart from staging laparoscopy being the essential step in the diagnosis and assessment of resectability.

\section{Results}

The included patients had a mean age of 67 (Range 55-72) years, with male patients being twice as many as female patients. The diagnoses after the systemic workups were as follows: duodenal adenocarcinoma (Four cases), pancreatic head cancer (15 cases), and distal bile duct adenocarcinoma (Nine cases), as can be seen in (Fig. 1).

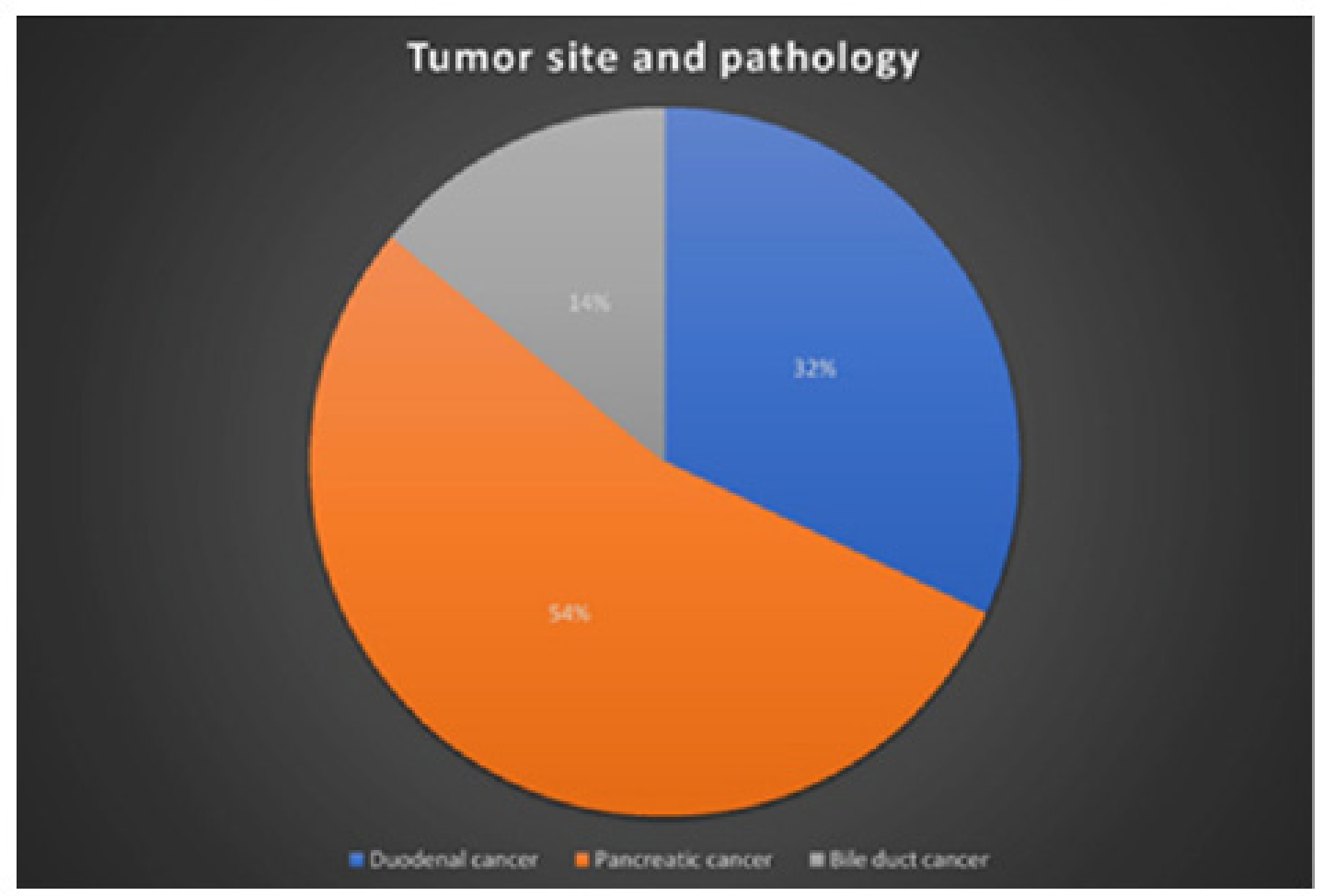

Fig 1: Tumor site and pathology.

In group $A$, six cases were diagnosed with advanced cancers, four cases had a tumor encasement $>180^{\circ}$ of the vascular arteries at the tumor bed, and two cases had liver seeds. These patients were referred to the palliative team for the initiation of chemotherapy or supportive treatment. Abdominal exploration was offered to the remaining eight cases. Moreover, three cases were understaged following CT, whereas two cases were found to have small liver metastasis. One case had malignant ascites confirmed by intraoperative aspiration cytology, which was missed during $\mathrm{CT}$.

Diagnostic laparoscopy in group B was done routinely, which subsequently confirmed the diagnosis in $78 \%(11 / 14)$ of the patients. Moreover, laparoscopy identified small liver seeds in two patients and malignant ascites in one patient, which were missed during CT (3/14). The postlaparoscopy staging in those three cases was upgraded to stage 4, which prevented unnecessary laparotomy. Moreover, one patient who was diagnosed with a resectable tumor after both MDCT and staging laparoscopy was found to have stage 3 pancreatic cancer encasing $>180^{\circ}$ of the SMA, which prompted an intraoperative decision of irresectability after unnecessary laparotomy.

Our finding showed that group A, where CT was the mainstay for staging, had a sensitivity and accuracy of $100 \%$ for identifying locally advanced and irresectable cancers as can be seen in (Fig. 2). CT was able to show clearly that the tumor had $>180^{\circ}$ of contact with the arterial vasculature in four cases. CT was also able to diagnose liver metastasis in the other two cases. The current study confirmed that MDCT had a positive predictive value of $100 \%$ for diagnosing irresectable tumors. However, its mean predictive value and accuracy dropped to $62.5 \%$ and $37.5 \%$ when confirming resectability, respectively. MDCT missed findings in three cases, which lead to underdiagnosis and mismanagement. 


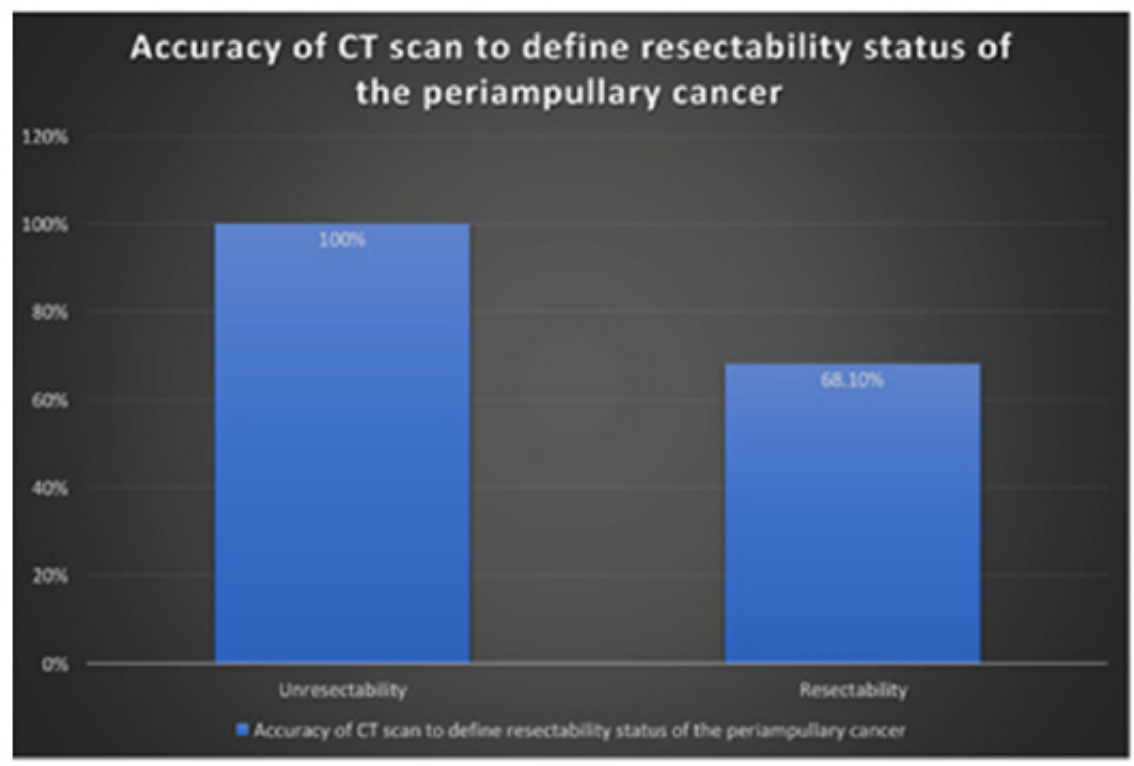

Fig 2: Accuracy of MDCT to define resectability status of peri-ampullary carcinoma.

In group $\mathrm{B}, \mathrm{MDCT}$ was able to successfully stage periampullary carcinoma in $10 / 14$ case with $71 \%$ accuracy and a false-negative percentage of $28.5 \%$. Three out of four cases were underdiagnosed during CT and were later confirmed by the diagnostic keyhole procedure, which found additional signs of irresectability (two patients had two small occult liver metastasis, whereas one patient had malignant peritoneal fluid). These patients were subsequently saved from a nonindicated exploration laparotomy and its associated morbidities and delay in systemic therapy. The camera was able to detect stage 3 pancreatic head cancer in one patient who underwent an unnecessary laparotomy. Therefore, MDCT had a mean predictive value of $71 \%$ in group
B, which increased to $79 \%$ after adding the camera to the routine workup.

The current study found that MDCT had a sensitivity and accuracy of $68.1 \%$ in resectable cancers, as can be seen in (Figure 2), and an overall accuracy of $75 \%$ in staging periampullary cancers after including irresectable cancers. The overall accuracy increased to $96.4 \%$ after adding staging laparoscopy to the diagnostic modalities. , as can be seen in (Fig. 3). CT had a false-negative rate of $21.4 \%$ $(3 / 14)$ and $28.5 \%(4 / 14)$ in groups $A$ and $B$, respectively, with an overall false-negative rate of $25 \%$. After adding the keyhole procedure, the falsenegative rate decreased to $7.1 \%(1 / 14)$.

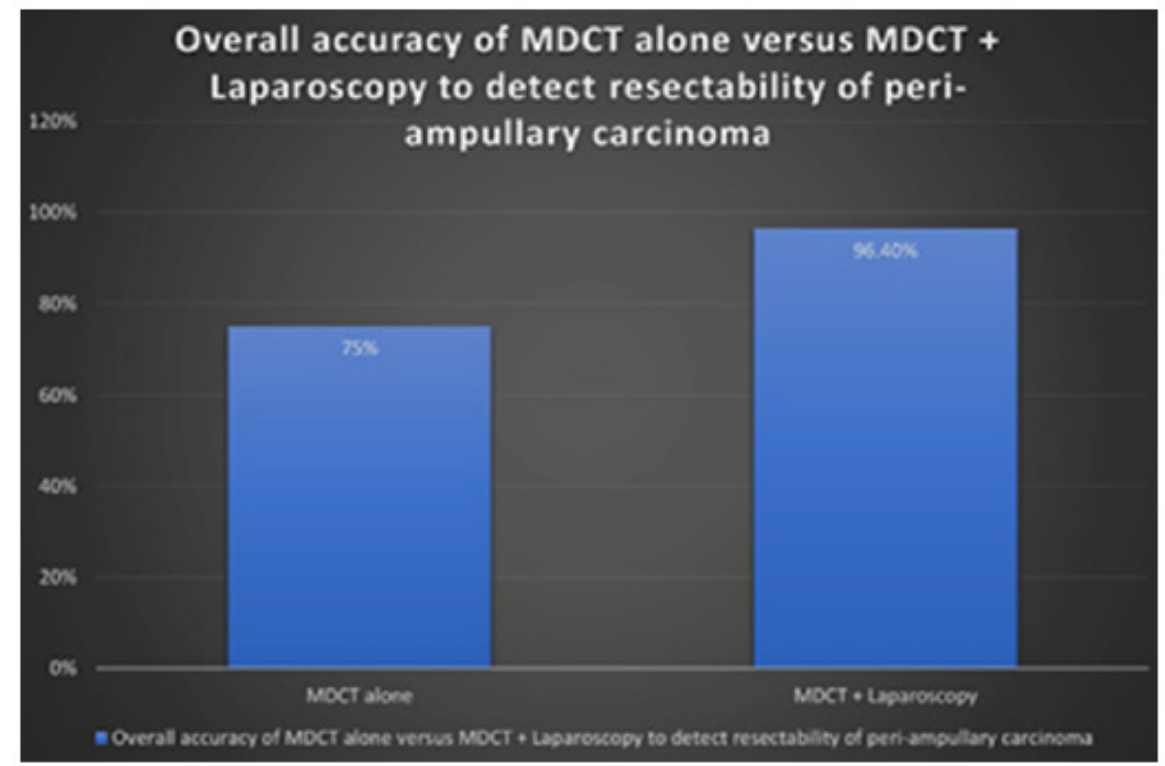

Fig 3: Overall accuracy of MDCT alone versus MDCT + laparoscopy to detect resectability. of peri-ampullary carcinoma. 


\section{Discussion}

Tertiary centers have utilized the CT pancreatic protocol as the main modality for staging periampullary pancreatic cancers. Given that MDCT can acquire isotropic volume information along the patient's longitudinal axis, this approach has been considered better than conventional abdominal CT. Moreover, MDCT produces $0.1-\mathrm{mm}$ thick slices, which can then be reconstructed to multiplanar sections of desired thickness or plane without losing any information or disrupting the anatomical layout. The three-dimensional ability can depict the relationship between the growth and the vascular structures adherent to it. An iodinated contrast medium is injected at many phases, thereby providing a dynamic enhancement pattern. The pancreatic phase (I.e., $30 \mathrm{~s}$ after dye injection) is the median balanced phase between the arterial (15 s) phase and the portal venous (>40 s) phase. The pancreatic phase has been shown to be highly sensitive for cancer detection. ${ }^{4}$ The portal window is when liver metastases can be visualized. The main difference between normal pancreatic tissue and neoplastic growth occurs at the pancreatic phase of the scan. Moreover, the degree of tumor abutment to the surrounding venous vessels is best assessed during this time. Accordingly, 3-mm images are captured at 20,40, and $65 \mathrm{~s}$, whereas delayed scans are captured at $120 \mathrm{~s}$ to avoid confusing liver metastasis with hemangiomas. ${ }^{5-8}$

Periampullary pancreatic neoplasms are a heterogeneous group of unrelated pathologies. MDCT helps map the local growth and complications such as tumor obstruction and nodal or distant spread. This group of pathologies shares low dye uptakes in both the arterial and pancreatic phases. Notably, ampulla of Vater cancers can be observed early with the double-duct sign, whereas duodenal cancers are usually observed at the later stages with annular narrowed segment and proximal dilatation. Gastrointestinal stromal tumors are enhanced homogenously in films. Malignant villous adenomas are hyper-enhancing lesions with pancreatic invasion at the arterial phase. Central attenuation resulting from central necrosis is a pathognomonic sign of metastatic lymph nodes. Pancreatic adenocarcinomas appear as a focal hypoattenuating mass during the pancreatic phase, with smooth dilation of the upstream biliopancreatic duct systems and a sharp cutoff at the mass level. Other findings demonstrated hypoattenuating cystic lesions with honeycomb shapes in the portal phase. Intraductal papillary mucinous neoplasms and neuroendocrine tumors (neuroendocrine tumors) can also be observed at the periampullary region. Cholangioadenorcinoma at the distal bile duct is another type of pathology that can originate from the biliary epithelium near the sphincter of Oddi. ${ }^{8-11}$
Over two-thirds of the patients diagnosed with curative tumors were found to be irresectable intraoperatively or have poor postoperative outcomes. The criteria for resectability included vascular proximity and detection of distant spread. Thearterial structures thatrequire careful assessment include the celiac artery, SMA with the first jejunal branch, and aorta. A single artery with $<180^{\circ}$ of encasement, no abnormal anatomy, reconstructable length, and no bifurcation involvement are all signs of borderline resectability. Tumor growth greater or less than $180^{\circ}$ with the superior mesenteric vein or portal vein, without luminal thrombosis or marked distortion, is considered borderline. The mean predictive value for irresectability and resectability is $90 \%-100 \%$ and $45 \%-79 \%$, respectively. At best, positron emission tomography can detect distant metastases with an average sensitivity of approximately $61 \%{ }^{3,5,10}$

In our study, MDCT was able to confirm irresectability in $100 \%$ of the cases, with the accuracy dropping to $68.1 \%$ when staging borderline cancers. However, MDCT failed to identify cases with occult liver metastasis (4/22, 18\%) and malignant ascites $(2 / 22,9 \%)$ and missed the presence of locally advanced cancer in one case (5\%). Thus, MDCT had an overall accuracy of $75 \%$ for staging periampullary neoplasms, which improved to $92.5 \%$ after adding diagnostic laparoscopy to the staging system. Falsenegative incidences were $21 \%$ and $7 \%$ in groups $A$ and $B$, respectively, with an overall incidence of $14.2 \%$. The mean predictive value of CT was $100 \%$ and $68.1 \%$ for irresectable and resectable cancers, respectively.

Diagnostic laparoscopy is a minimally invasive exploration of the abdomen that requires three $5-\mathrm{mm}$ ports to access the abdomen. Including this 30-min procedure can help prevent needless laparotomies and augment CT sensitivity for staging borderline cancer. Moreover, this procedure can detect occult liver spread or minimal malignant effusion or peritoneal seeds commonly missed by imaging, which could lead to the understaging of the neoplasm. Others studies have investigated the addition of intraoperative laparoscopic ultrasound to examine the posterior arteries and degree of cancer abutment. ${ }^{1,2,12,13}$

\section{Conclusions}

MDCT has been the cornerstone noninvasive modality for staging periampullary pancreatic tumors considering its sensitivity and accuracy, both of which unfortunately decrease markedly in borderline cancers. The scan can easily overlook occult liver seeds and mild malignant ascites, thereby causing understaging and unnecessary attempts for curative surgery. Our findings showed that adding other diagnostic tools can improve the accuracy of MDCT. Staging laparoscopy and 
positron emission tomography can be beneficial for patients with equivocal findings, with some centers advocating for the routine use of laparoscopy in staging pancreatic and periampullary cancers.

\section{References}

1. Karabicak I, Satoi $S$, Yanagimoto $H$, et al: Risk factors for latent distant organ metastasis detected by staging laparoscopy in patients with radiologically defined locally advanced pancreatic ductal adenocarcinoma. J Hepatobiliary Pancreat Sci. 2016; 23: 750-5. 10.1002/jhbp.408.

2. Bemelman WA, de Wit LT, Van Delden OM, et al: Diagnostic laparoscopy combined with laparoscopic ultrasonography in staging of cancer of the pancreatic head region. Br J Surg. 1995; 82: 820-4. 10.1002/bjs.1800821248.

3. Sahani DV, Shah ZK, Catalano OA, et al.: Radiology of pancreatic adenocarcinoma: current status of imaging. $J$ Gastroenterol Hepatol. 2008; 23: 23-33. 10.1111/j.14401746.2007.05117.x.

4. Computed Tomography (CT) Scan of the Pancreas. (2021). Accessed: 14/10/2021: https://www.hopkinsmedicine.org/health/ treatment-tests-and-therapies/computedtomography-ct-scan-of-the-pancreas.

5. Pietryga JA, Morgan DE: Imaging preoperatively for pancreatic adenocarcinoma. J Gastrointest Oncol. 2015; 6: 343-57.

6. Allen VB, Gurusamy KS, Takwoingi $Y$, et al: Diagnostic accuracy of laparoscopy following computed tomography (CT) scanning for assessing the resectability with curative intent in pancreatic and periampullary cancer. Cochrane
Database Syst Rev. 2016; 7: 009323-10.

7. Agarwal AK, Kalayarasan $\mathrm{R}$, Javed $\mathrm{A}$, et al: The role of staging laparoscopy in primary gall bladder cancer - an analysis of 409 patients: a prospective study to evaluate the role of staging laparoscopy in the management of gallbladder cancer. Ann Surg. 2013; 258: 318-23. 10.1097/ sla.0b013e318271497e.

8. Al-Hawary MM, Kaza RK, Wasnik AP, et al: Staging of pancreatic cancer: role of imaging. Semin Roentgenol. 2013; 48: 245-52. 10.1102/1470-7330.2013.9020.

9. Arumugam P, Balarajah V, Watt J, et al: Role of laparoscopy in hepatobiliary malignancies. Indian J Med Res. 2016; 143: 414-9. 10.4103/0971-5916.184300.

10. Matrisian LM, Aizenberg $R$, Rosenzweig A: Pancreatic Cancer Action Network: The alarming rise of pancreatic cancer deaths in the United States: why we need to stem the tide today. 120202.

11. Al-Hawary MM, Francis IR, Chari ST, et al: Pancreatic ductal adenocarcinoma radiology reporting template: consensus statement of the Society of Abdominal Radiology and the American Pancreatic Association. Radiology. 2014; 270: 248-60. 10.1148/radiol.13131184.

12. Miki $Y$, Tokunaga $M$, Tanizawa $Y$, et al: Staging laparoscopy for patients with cM0, type 4, and large type 3 gastric cancer. World J Surg. 2015; 39: 2742-7. 10.1007/s00268-015-3144-z.

13. Königsrainer I, Zieker D, Symons S, et al: Do patient- and tumor-related factors predict the peritoneal spread of pancreatic adenocarcinoma?. Surg Today. 2014; 44: 260-3. 10.1007/s00595-013-0546-0. 